(2) Open Access Full Text Article

\title{
How many ELNs are optimal for breast cancer patients with more than three PLNs who underwent MRM? A large population-based study
}

This article was published in the following Dove Press journal:

OncoTargets and Therapy

Xiaohui Wang'
Changbin $\mathrm{Ji}^{2}$
Huiying $\mathrm{Chi}^{3}$
Haiyong Wang

'Research Service Office, Shandong Liaocheng People's Hospital, Liaocheng, China; ${ }^{2}$ Orthopedics Department, Shandong Liaocheng People's Hospital, Liaocheng, China; ${ }^{3}$ Shanghai Geriatrics Institute of Traditional Chinese Medicine, Shanghai, China; ${ }^{4}$ Department of Internal Medicine-Oncology, Shandong Cancer Hospital and Institute, Shandong Cancer Hospital affiliated to Shandong University, Shandong Academy of Medical Sciences, Jinan, China
Correspondence: Haiyong Wang Department of Internal MedicineOncology, Shandong Cancer Hospital and Institute, Shandong Cancer Hospital affiliated to Shandong University, Shandong Academy of Medical Sciences, 440 Jiyan Road, Huaiyin District, Jinan 2501 I7, China

Tel +86053187984777

Fax +8653187984079

Email wanghaiyong6688@I26.com
Background: Few studies have focused on the optimal threshold of examed lymph nodes (ELNs) for breast cancer patients with more than three positive lymph nodes after modified radical mastectomy.

Materials and methods: The X-tile and the minimum $P$-value models were applied to determine the optimal threshold. Cox proportional hazard analysis was used to analyze the cancer-specific survival and perform subgroup analysis.

Results: The results showed that 12 ELNs was the optimal threshold for these patients, and the patients with $>12$ ELNs had a better cancer-specific survival benefit compared with the patients with $<12$ ELNs $(P<0.001)$.

Conclusion: The number 12 can be selected as the optimal threshold of ELNs for breast cancer patients with $>3$ positive lymph nodes after modified radical mastectomy.

Keywords: breast cancer, mastectomy, ELNs, positive lymph nodes, X-tile

\section{Introduction}

Modified radical mastectomy (MRM) serves as the primary treatment strategy for early breast cancer patients. ${ }^{1,2}$ The status of local positive lymph nodes (PLNs) after MRM has been regarded as an important prognostic factor for patients with breast cancer..$^{3-5}$ Some lymph node parameters including the ratio of PLNs and the number of negative lymph nodes have been introduced and demonstrated to assume more powerful role in predicting survival prognosis for breast cancer patients..$^{6-9}$ In addition, the number of PLNs strongly associated with American Joint Committee on Cancer (AJCC) N stage is a critical factor to determine the strategies in adjuvant systemic therapy. ${ }^{10-13}$

Theoretically, more number of examed lymph nodes (ELNs) after MRM could increase the probability of PLNs, thus providing more accurate information on TNM stage and adjuvant therapy. However, it is worth noting that more number of ELNs is associated with an increased risk of adverse outcomes. ${ }^{14-16}$ Therefore, it is vitally important to find an optimal threshold of ELNs, so as to balance the two key clinical prognosis factors. It is a pity that few studies have focused on these issues of clinical significance, especially in recent years.

To address these unresolved issues, using the Surveillance, Epidemiology, and End Results (SEER)-registered database based on a large sample size and high-quality population-based US cancer registries we focused on the breast cancer patients with more than three PLNs undergoing MRM and further confirmed the relationship between 
the number of ELN and cancer-specific survival (CSS). Importantly, multiple models were applied to determine the optimal threshold for ELN count.

\section{Materials and methods}

\section{Patient selection}

The SEER Cancer Statistics Review ${ }^{28}$ is published annually by the Data Analysis and Interpretation Branch of the National Cancer Institute, Bethesda, MD, USA. A total of 18 population-based cancer registries in the USA were included in the current SEER database. ${ }^{17}$ We used SEER*Stat software 8.3.2 to screen the appropriate patients with breast cancer between 2004 and 2009. Patients with the following criteria were included: female with confirmed age and only one primary tumor; the diagnosis was confirmed microscopically; and active follow-up. In addition, the breast cancer patients should have received MRM and should be with more than three PLNs removed. The breast cancer patients who underwent conserving surgery were not included in our study. All the breast cancer patients included in our study had conducted the MRM and did not have the sentinel lymph node biopsy performed. Breast cancer patients lacking information on the subgroup variables including age, race, AJCC T stage, grade, and the number of ELNs were all excluded. We also excluded the patients lacking information on the cause of death and survival months.

\section{Ethics statement}

As we have previously mentioned, ${ }^{18}$ this study was mainly based on the SEER database and was conducted in compliance with the Helsinki Declaration. We obtained permission to access the files of SEER program research data and the reference number is 11304-November 2015. Informed consent was not required because personal identifying information was not involved. This study was approved by the ethics committee of the Shandong Cancer Hospital affiliated to Shandong University.

\section{Statistical analysis}

We mainly analyzed the following variables: age, race, grade, AJCC T stage, and the number of ELNs. We obtained CSS from the SEER database and identified it as the terminal point of the patients. CSS is a survival measure representing survival of a specified cancer of death in the absence of other causes of death. ${ }^{18}$ The influence of ELN numbers on CSS was measured by Cox proportional hazard. We determined the optimal threshold for the number of ELNs by the X-tile mode. Then, the optimal threshold was validated by using the minimum $P$-value model. Based on the optimal threshold, subgroup analyses of the CSS were conducted by Cox proportional hazard analysis based on different variables and presented on the forest plot.

As we have previously mentioned, ${ }^{18}$ all statistical tests were two-sided and a $P<0.05$ was considered statistically significant. The statistical software SPSS 18.0 (SPSS Inc., Chicago, IL, USA) was used for all data analyses.

\section{Results}

\section{Patient demographics}

A cohort of 9,297 female breast cancer patients was selected between 2004 and 2009 from SEER database. They were followed up for a consecutive 119 months. Generally, the minimum number of ELNs among these female breast cancer patients was 4 and the maximum number of ELNs was 86 . The proportion of white-raced patients and diagnosed at the age of $\geq 50$ years was $78.0 \%$ and $66.2 \%$, respectively, accounting for a large proportion among the female breast cancer patients. The ratio of breast cancer patients at Grade I, II, and III was $6.3 \%, 37.4 \%$, and $56.3 \%$, respectively. Our results also demonstrated that $61.7 \%$ of the patients were diagnosed at AJCC stage T1-2. Their clinical characteristics are presented in Table 1.

Table I Characteristics of patients with more than three PLNs who underwent MRM and were screened from the SEER database

\begin{tabular}{ll}
\hline Variables & Number (\%) \\
\hline Total & $9,297(100)$ \\
Number of ELNs & $4-86$ \\
Age, years & \\
$\quad<50$ & $3,146(33.8)$ \\
$\geq 50$ & $6,151(66.2)$ \\
Race & \\
White & $7,253(78.0)$ \\
Black & $1,229(13.2)$ \\
Others & $815(8.8)$ \\
Grade & \\
I & $588(6.3)$ \\
II & $3,473(37.4)$ \\
III & $5,236(56.3)$ \\
T stage & \\
TI & $1,459(15.7)$ \\
T2 & $4,273(46.0)$ \\
T3 & 2,10 I (22.6) \\
T4 & $1,464(15.7)$ \\
\hline A
\end{tabular}

Abbreviations: ELNs, examed lymph nodes; MRM, modified radical mastectomy; PLNs, positive lymph nodes; SEER, Surveillance, Epidemiology, and End Results. 


\section{The relationship between the number of ELNs and PLNs}

Generally, more numbers of ELNs after MRM may increase the probability of PLNs. Pearson correlation analysis was applied to confirm the relationship. The results showed a positive significant relationship between the number of ELNs and PLNs $(P<0.001$; Figure 1).

\section{Number of ELNs and CSS}

The association between the ELN count (as a continuous variable) and CSS was measured by Cox proportional hazard. As we expected, the number of ELNs was found to be of prognostic significance on CSS (hazard ratio [HR]: 0.992, 95\% CI: 0.987-0.997, $P=0.001$; Table 2).

\section{Determining the optimal threshold for the number of ELNs}

Next, we determined the optimal threshold for the number of ELNs by the X-tile mode. The optimal threshold was found to be 12 (Figure 2A and B). Then, the CSS curve was generated by Kaplan-Meier analyses, and the survival prognosis was evaluated by $\log$ rank $\chi^{2}$. Survival curves revealed that the patients an ELN number $>12$ had a better CSS rate $(P<0.001$; Figure 2C).

\section{Validation of the optimal threshold of ELNs}

To verify the validity of 12 as the optimal threshold of ELNs, we further analyzed individual number of ELNs from 5 to 17 using the log-rank $\chi^{2}$. The 5-year CSS was calculated based on different numbers of ELNs, respectively. The minimum $P$-value model was applied to validate the optimal threshold. The results showed that the patients with $>12$ ELNs count had a maximum log-rank $\chi^{2}$ value compared with the patients

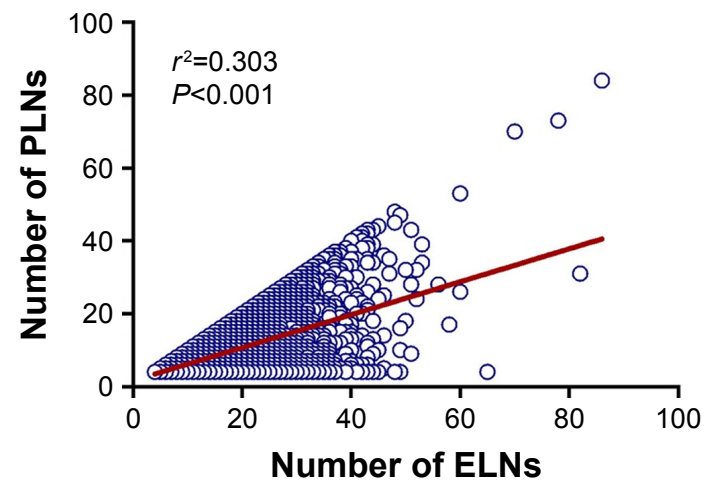

Figure I The relationship between the number of ELNs and PLNs demonstrated by Pearson correlation analysis $(P<0.00 \mathrm{I})$.

Abbreviations: ELNs, examed lymph nodes; PLNs, positive lymph nodes.
Table 2 The influence of different variables on CSS for breast cancer patients with more than three PLNs who underwent MRM analyzed by Cox proportional hazard model

\begin{tabular}{|c|c|c|c|c|}
\hline \multirow[t]{2}{*}{ Variables } & \multicolumn{2}{|c|}{$\begin{array}{l}\text { Univariate } \\
\text { analysis }\end{array}$} & \multicolumn{2}{|l|}{$\begin{array}{l}\text { Multivariate } \\
\text { analysis }\end{array}$} \\
\hline & Wald $\chi^{2}$ & $P$-value & HR (95\% CI) & $P$-value \\
\hline Number of ELNs & 13.782 & $<0.001$ & $0.992(0.987-0.997)$ & 0.001 \\
\hline Age, years & 14.355 & $<0.001$ & & $<0.001$ \\
\hline$<50$ & & & Reference & \\
\hline$\geq 50$ & & & $1.311(1.214-1.415)$ & $<0.001$ \\
\hline $\begin{array}{l}\text { Race } \\
\text { White } \\
\text { Black } \\
\text { Others }\end{array}$ & 0.046 & 0.830 & Not included & \\
\hline Grade & 268.909 & $<0.001$ & & $<0.001$ \\
\hline I & & & Reference & \\
\hline II & & & 1.677 (1.360-2.067) & $<0.001$ \\
\hline III & & & $2.838(2.315-3.480)$ & $<0.001$ \\
\hline T stage & 447.965 & $<0.001$ & & $<0.001$ \\
\hline TI & & & Reference & \\
\hline $\mathrm{T} 2$ & & & $1.584(1.393-1.80 I)$ & $<0.001$ \\
\hline T3 & & & $2.327(2.033-2.663)$ & $<0.001$ \\
\hline $\mathrm{T} 4$ & & & $3.208(2.797-3.679)$ & $<0.001$ \\
\hline
\end{tabular}

Abbreviations: CSS, cancer-specific survival; ELNs, examed lymph nodes; HR, hazard ratio; MRM, modified radical mastectomy; PLNs, positive lymph nodes.

with $\leq 12$ ELNs $\left(\chi^{2}=28.85, P<0.001\right.$; Table 3$)$. Therefore, 12 was validated as the optimal threshold for these patients. In addition, the 5-year CSS rate of the breast cancer patients with $>12$ ELNs was $73.3 \%$ (Table 3). Also, the 5-year CSS rate of the breast cancer patients with $\leq 12$ ELNs was $68.3 \%$ (Table 3).

\section{Subgroup analysis for CSS}

After adjusting for other prognostic factors including age, race, grade, and AJCC T stage, we analyzed the CSS of subgroup variables based on the optimal threshold. The specific results were presented on the forest plot. The results demonstrated that all the subgroup variables including age $<50$, age $\geq 50$, white race, grade II and III, and AJCC T2-4, the number of ELNs $>12$ showed significant CSS benefits (age <50: HR: 0.851, 95\% CI: 0.742-0.977, $P=0.022$; age $\geq 50$ : HR: $0.839,95 \%$ CI: $0.768-0.917, P<0.001$; white race: HR: $0.842,95 \%$ CI: $0.772-0.917, P<0.001$; grade II: HR: $0.825,95 \%$ CI: $0.720-0.946, P=0.006$; grade III: HR: 0.843, 95\% CI: 0.770-0.924, $P<0.001$; AJCC T2: HR: 0.862, 95\% CI: 0.766-0.971, $P=0.014$; AJCC T3: HR: 0.860, 95\% CI: $0.741-0.999, P=0.048$; AJCC T4: HR: $0.815,95 \% \mathrm{CI}$ : 0.704-0.944, $P=0.006$; Figure 3).

\section{Discussion}

The number of PLNs has been demonstrated to play an important role in determining adjuvant therapy by providing 

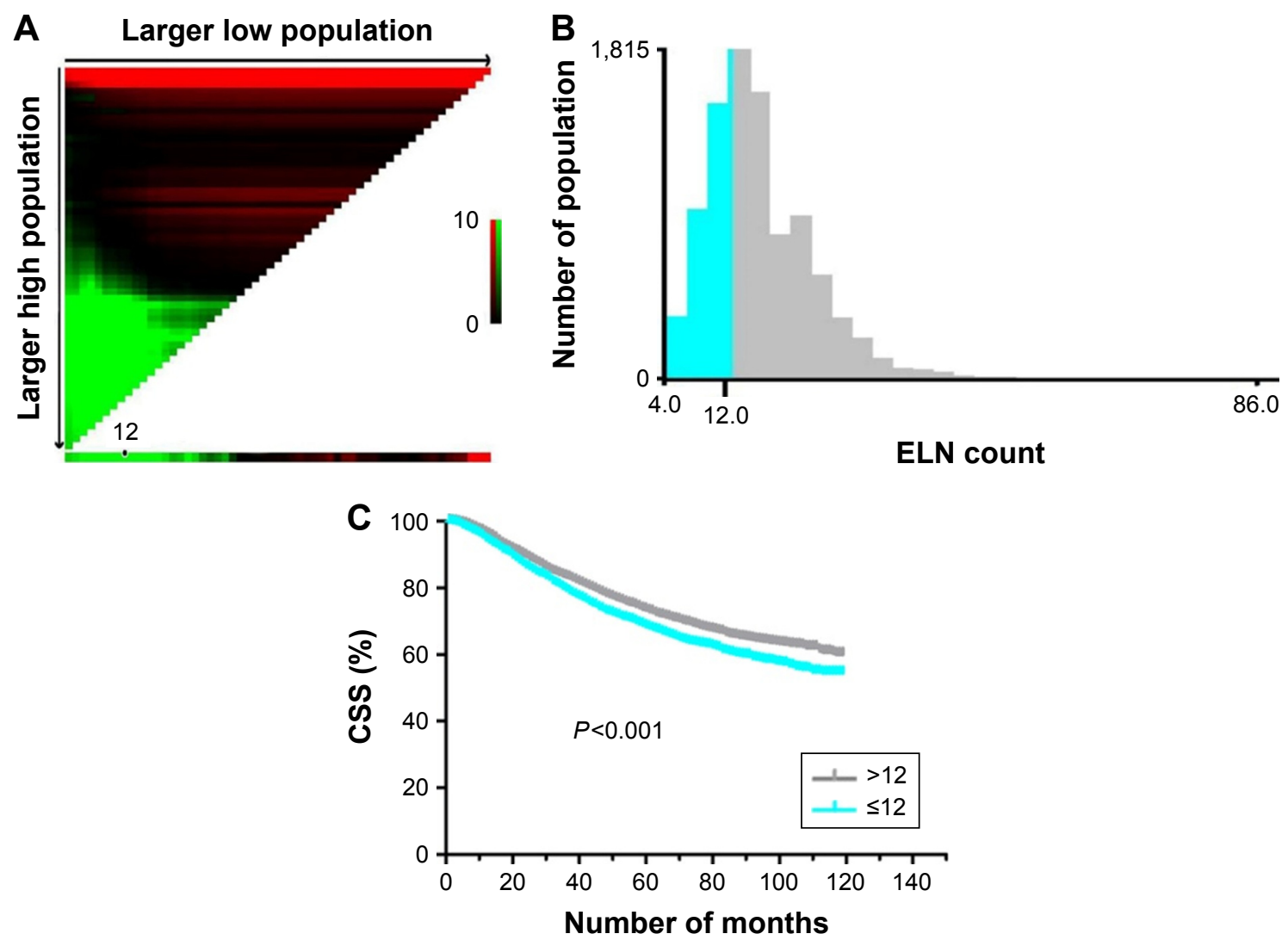

Figure 2 The optimal threshold of ELNs screened by the $X$-tile model.

Notes: (A) X-tile plots based on the number of ELNs. (B) The optimal cut-off point stressed by the gray and green panels. (C) The CSS curves based on the threshold $(P<0.001)$.

Abbreviations: CSS, cancer-specific survival; ELNs, examed lymph nodes.

accurate information on $\mathrm{N}$ stage of TNM stage for breast cancer patients..$^{10-13}$ The number of PLNs has been applied by the AJCC staging system to classify the breast cancer patients with 1-3 PLNs as pN1 stage, 4-9 PLNs as pN2 stage, and $\geq 10$ PLNs as pN3 stage. ${ }^{19,20}$ In fact, National Comprehensive Cancer Network and several other studies have regarded radiotherapy after MRM as standard treatment only for breast cancer patients with more than three PLNs. ${ }^{12,21}$ Therefore, the number of three has been viewed a key point to determine adjuvant therapy for patients with breast cancer after MRM. Based on these scenarios, we only screened the appropriate breast cancer patients with more than three PLNs undergoing MRM. Theoretically, the number of PLNs depends on the number of ELNs. In other words, more numbers of ELNs would increase the probability of PLNs. As we expected, the relationship between the number of ELNs and PLNs has been reflected in Figure 1.

From the point of view of adjuvant treatment, more number of ELNs could reduce the proportion of understaged patients and provide more breast cancer patients with adjuvant therapy after MRM. Several studies have also shown that the number of ELNs has an intimate correlation with the survival benefit of patients..$^{22,23}$ However, from the point of view of adverse outcomes with lymph node surgery, more number of ELNs could inevitably lead to more complications including pain, infection, seroma formation, restricted shoulder mobility, and sometimes even lymphedema. ${ }^{14-16}$ Importantly, more number of ELNs will destroy the immune microenvironment and affect the organism's immunity function negatively. ${ }^{24,25}$ Therefore, it is very important to strike a balance between increasing the detection rate of PLNs and reducing the incidence of complications. However, few studies have focused on the key clinical problem. In our study, using the SEER database, patients who underwent MRM and with more than three PLNs were only enrolled, and we regarded CSS as our main end point. As previously described, ${ }^{18}$ the X-tile model was used to determine the optimal threshold of ELNs. In fact, the X-tile plots can provide a single, global assessment of every possible way of dividing a population into low- and high-level marker expression. In addition, the X-tile can 
Table 3 Univariate analysis to evaluate the influence of different cut-off points on CSS for breast cancer patients with more than PLNs who underwent MRM

\begin{tabular}{|c|c|c|c|c|}
\hline Cut-off & Number & $\begin{array}{l}\text { 5-year } \\
\text { CSS }\end{array}$ & $\begin{array}{l}\log \\
\operatorname{rank} \chi^{2}\end{array}$ & $P$-value \\
\hline$\leq 5$ & 165 & 60.8 & 8.47 & 0.004 \\
\hline$>5$ & 9,132 & 72.0 & & \\
\hline$\leq 6$ & 342 & 62.1 & 15.10 & $<0.001$ \\
\hline$>6$ & 8,955 & 72.2 & & \\
\hline$\leq 7$ & 570 & 63.9 & 22.40 & $<0.001$ \\
\hline$>7$ & 8,727 & 72.3 & & \\
\hline$\leq 8$ & 848 & 65.1 & 20.63 & $<0.001$ \\
\hline$>8$ & 8,449 & 72.5 & & \\
\hline$\leq 9$ & 1,276 & 66.6 & 18.40 & $<0.001$ \\
\hline$>9$ & 8,021 & 72.6 & & \\
\hline$\leq 10$ & $|, 73|$ & 67.1 & 24.43 & $<0.001$ \\
\hline$>10$ & 7,566 & 72.9 & & \\
\hline$\leq 11$ & 2,246 & 67.8 & 26.03 & $<0.001$ \\
\hline$>11$ & 7,051 & 73.1 & & \\
\hline$\leq 12$ & 2,792 & 68.3 & 28.85 & $<0.00 \mathrm{I}$ \\
\hline$>12$ & 6,505 & 73.3 & & \\
\hline$\leq 13$ & 3,398 & 69.0 & 23.19 & $<0.00 \mathrm{I}$ \\
\hline$>13$ & 5,899 & 73.4 & & \\
\hline$\leq 14$ & 3,981 & 69.7 & 21.55 & $<0.001$ \\
\hline$>14$ & 5,316 & 73.4 & & \\
\hline$\leq 15$ & 4,607 & 70.6 & 9.92 & $<0.001$ \\
\hline$>15$ & 4,690 & 73.1 & & \\
\hline$\leq 16$ & 5,173 & 70.6 & 12.19 & $<0.001$ \\
\hline$>16$ & 4,124 & 73.4 & & \\
\hline$\leq 17$ & 5,719 & 70.8 & 8.81 & 0.003 \\
\hline$>17$ & 3,578 & 73.5 & & \\
\hline
\end{tabular}

Abbreviations: CSS, cancer-specific survival; MRM, modified radical mastectomy; PLNs, positive lymph nodes. produce corrected $P$-values using several Monte Carlo simulations: cross-validation takes our dataset, randomly splits it into two halves, and finds the optimal cut-off point of one half and then divides the other half according to this cut-off point. Then, it finds the optimal cut-off point of the second half and similarly divides the first. ${ }^{26}$ The results showed that the number 12 can be regarded as the optimal threshold for these breast cancer patients. In order to ensure the accuracy of the results, another model named minimum $P$-value method applied in others studies ${ }^{9,27}$ was used to further validate the feasibility of using 12 as the optimal threshold for ELN count. Our finding may give some suggestions and references to surgical specialists or pathologists to determine the appropriate number of lymph nodes to be dissected.

\section{Strength and limitations}

To our knowledge, this study is currently the largest study focusing on such a clinical problem using huge and realistic data. We sought to emphasize that breast cancer patients with more than three PLNs after MRM should reasonably determine the number of ELNs based on the survival prognosis and adverse reaction. Undeniably, this study also has several limitations. First, some important factors that affected prognosis, such as adjuvant therapy, estrogen receptor/progesterone receptor status, HER-2, and so on, were not included in our study. In fact, the $\mathrm{X}$-tile and minimum $P$-value model were applied to determine the optimal threshold for breast cancer patients with more than three PLNs after MRM in our

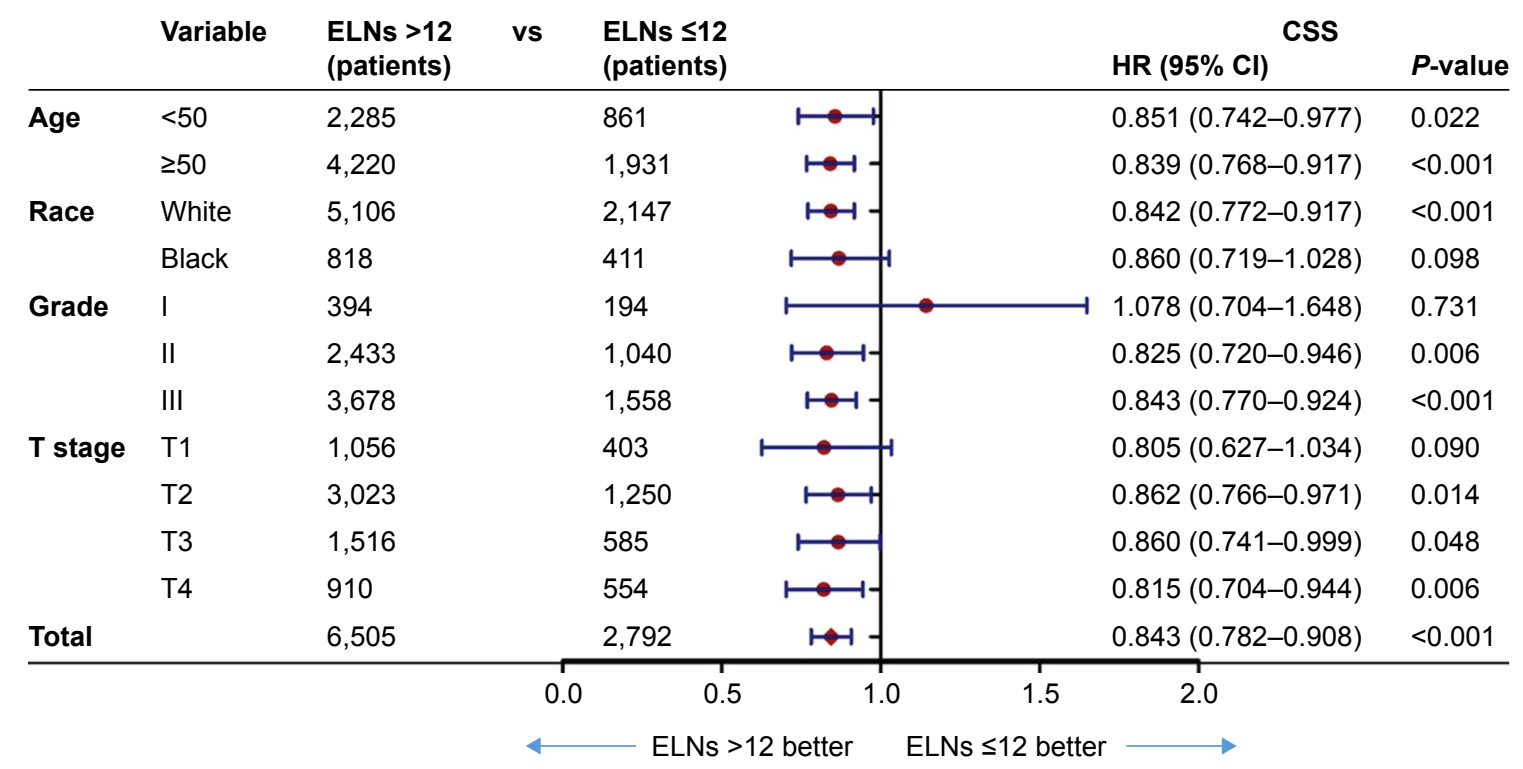

Figure 3 The forest plot of the number of ELNs on CSS based on all the subgroup variables conducted by the Cox proportional hazard model. Abbreviations: CSS, cancer-specific survival; ELNs, examed lymph nodes; HR, hazard ratio. 
study. The variables including the number of ELNs, survival month, and CSS status were the three key factors to determine the optimal threshold. Although the important factors affecting prognosis, such as adjuvant therapy, estrogen receptor/ progesterone receptor status, HER-2, and so on, were not included in our study, the final conclusion is largely unaffected. Of course, sufficient subgroup analyses would render our research more individualized and instructive.

In conclusion, our results showed that the number of 12 can be selected as the optimal threshold for ELNs for these patients. In addition, in clinical practice, we should determine the best ELN number for patients to conduct individual lymphadenectomies taking into account other factors and based on our results. Therefore, our results do not mean that a new lymphadenectomy will be performed or the lymphadenectomy is not done if the patients have $<12$ ELNs after MRM. Of course, further randomized controlled study and sufficient subgroup analyses are needed to validate our conclusion.

\section{Acknowledgments}

This study was supported jointly by the National Natural Science Foundation of China (No. 81603348), China Postdoctoral fund (No. 21300075311104), and Shandong postdoctoral innovation special fund (No. 201602012).

\section{Disclosure}

The authors report no conflicts of interest in this work.

\section{References}

1. Cotlar AM, Dubose JJ, Rose DM. History of surgery for breast cancer: radical to the sublime. Curr Surg. 2003;60(3):329-337.

2. Loukas M, Tubbs RS, Mirzayan N, Shirak M, Steinberg A, Shoja MM. The history of mastectomy. Am Surg. 2011;77(5):566-571.

3. Carter CL, Allen C, Henson DE. Relation of tumor size, lymph node status, and survival in 24,740 breast cancer cases. Cancer. 1989;63(1): 181-187.

4. Mwakigonja AR, Rabiel H, Mbembati NA, Lema LE. The pattern of prognostic and risk indicators among women with breast cancer undergoing modified radical mastectomy in Dar es Salaam, Tanzania. Infect Agent Cancer. 2016;11:28.

5. Vinh-Hung V, Cserni G, Burzykowski T, van de Steene J, Voordeckers M, Storme G. Effect of the number of uninvolved nodes on survival in early breast cancer. Oncol Rep. 2003;10(2):363-368.

6. Chagpar AB, Camp RL, Rimm DL. Lymph node ratio should be considered for incorporation into staging for breast cancer. Ann Surg Oncol. 2011;18(11):3143-3148.

7. Dings PJ, Elferink MA, Strobbe LJ, de Wilt JH. The prognostic value of lymph node ratio in node-positive breast cancer: a Dutch nationwide population-based study. Ann Surg Oncol. 2013;20(8):2607-2614.

8. Duraker N, Batı B, Caynak ZC, Demir D. Lymph node ratio may be supplementary to TNM nodal classification in node-positive breast carcinoma based on the results of 2,151 patients. World J Surg. 2013;37(6): 1241-1248.

9. Wang H, Zhang C, Kong L, Zhu H, Yu J. Better survival in PMRT of female breast cancer patients with $>5$ negative lymph nodes: A population-based study. Medicine (Baltimore). 2017;96(4):e5998.
10. Escobar PF, Patrick RJ, Rybicki LA, Weng DE, Crowe JP. The 2003 revised TNM staging system for breast cancer: results of stage reclassification on survival and future comparisons among stage groups. Ann Surg Oncol. 2007;14(1):143-147.

11. Wu SG, Chen Y, Sun JY, et al. Using the lymph nodal ratio to predict the risk of locoregional recurrence in lymph node-positive breast cancer patients treated with mastectomy without radiation therapy. Radiat Oncol. 2013;8:119.

12. Wang H, Kong L, Zhang C, Chen D, Zhu H, Yu J. Should all breast cancer patients with four or more positive lymph nodes who underwent modified radical mastectomy be treated with postoperative radiotherapy? A population-based study. Oncotarget. 2016;7(46):75492-75502.

13. Lin J, Li C, Zhang C, Shi F, Wang H. Postmastectomy radiation therapy for breast cancer patients with one to three positive lymph nodes: a propensity score matching analysis. Future Oncol. 2017;13(16):1395-1404.

14. Fleissig A, Fallowfield LJ, Langridge CI, et al. Post-operative arm morbidity and quality of life: results of the ALMANAC randomised trial comparing sentinel node biopsy with standard axillary treatment in the management of patients with early breast cancer. Breast Cancer Res Treat. 2006;95(3):279-293.

15. de Glas NA, Kiderlen M, Bastiaannet E, et al. Postoperative complications and survival of elderly breast cancer patients: a FOCUS study analysis. Breast Cancer Res Treat. 2013;138(2):561-569.

16. de Kanter AY, van Eijck CH, van Geel AN, et al. Multicentre study of ultrasonographically guided axillary node biopsy in patients with breast cancer. Br J Surg. 1999;86(11):1459-1462.

17. Surveillance, Epidemiology, and End Results (SEER) Program (www.seer.cancer.gov) SEER*Stat Database: Incidence - SEER 18 Regs Research Data + Hurricane Katrina Impacted Louisiana Cases, Nov 2015 Sub (1973-2013 varying) - Linked To County Attributes Total U.S., 1969-2014 Counties, National Cancer Institute, DCCPS, Surveillance Research Program, Surveillance Systems Branch, released April 2016, based on the November 2015 submission.

18. Chi H, Zhang C, Wang H, Wang Z. The appropriate number of ELNs for lymph node negative breast cancer patients underwent MRM: a population-based study. Oncotarget. 2017;8(39):65668-65676.

19. Singletary SE, Allred C, Ashley P, et al. Staging system for breast cancer: revisions for the 6th edition of the AJCC Cancer Staging Manual. Surg Clin North Am. 2003;83(4):803-819.

20. Amin MB, Edge S, Greene F, et al. AJCC Cancer Staging Manual. Springer; 2017. Available from: http://www.springer.com/us/book/ 9783319406176. Accessed February, 2017.

21. Clarke M, Collins R, Darby S, et al. Effects of radiotherapy and of differences in the extent of surgery for early breast cancer on local recurrence and 15-yeae survival: an overview of the randomized trials. Lancet. 2005;366(9503):2087-2106.

22. Xelsson CK, Mouridsen HT, Zedeler K. Axillary dissection of level I and II lymph nodes is important in breast cancer classification. The Danish Breast Cancer Cooperative Group (DBCG). Eur J Cancer. 1992; 28A(8-9):1415-1418.

23. Krag DN, Single RM. Breast cancer survival according to number of nodes removed. Ann Surg Oncol. 2003;10(10):1152-1159.

24. Camp RL, Rimm ED, Rimm DL. A high number of tumor-free lymph nodes from patients with lymph node negative breast carcinoma is associated with poor outcome. Cancer. 2000;88(1):108-113.

25. Schmidt M, Böhm D, von Törne C, et al. The humoral immune system has a key prognostic impact in node-negative breast cancer. Cancer Res. 2008;68(13):5405-5413.

26. Camp RL, Dolled-Filhart M, Rimm DL. X-tile: a new bio-informatics tool for biomarker assessment and outcome-based cut-point optimization. Clin Cancer Res. 2004;10(21):7252-7259.

27. Li Q, Zhuo C, Cai G, Li D, Liang L, Cai S. Increased number of negative lymph nodes is associated with improved cancer specific survival in pathological IIIB and IIIC rectal cancer treated with preoperative radiotherapy. Oncotarget. 2014;5(23):12459-12471.

28. The SEER Cancer Statistics Review [webpage on the Internet]. Available from: http://seer.cancer.gov/data/citation.html. Accessed April, 2017. 
OncoTargets and Therapy

\section{Publish your work in this journal}

OncoTargets and Therapy is an international, peer-reviewed, open access journal focusing on the pathological basis of all cancers, potential targets for therapy and treatment protocols employed to improve the management of cancer patients. The journal also focuses on the impact of management programs and new therapeutic agents and protocols on

patient perspectives such as quality of life, adherence and satisfaction. The manuscript management system is completely online and includes a very quick and fair peer-review system, which is all easy to use. Visit http://www.dovepress.com/testimonials.php to read real quotes from published authors.

Submit your manuscript here: http://www.dovepress.com/oncotargets-and-therapy-journal 